\title{
Maintaining Mobile Network Coverage Availability in Disturbance Scenarios
}

\author{
Joonas Säe and Jukka Lempiäinen \\ Department of Electronics and Communications Engineering, Tampere University of Technology, Tampere, Finland
}

Correspondence should be addressed to Joonas Säe; joonas.sae@tut.fi

Received 11 July 2016; Accepted 22 September 2016

Academic Editor: Ioannis Moscholios

Copyright ( $(2016$ J. Säe and J. Lempiäinen. This is an open access article distributed under the Creative Commons Attribution License, which permits unrestricted use, distribution, and reproduction in any medium, provided the original work is properly cited.

\begin{abstract}
Disturbance and disaster scenarios prevent the normal utilization of mobile networks. The aim of this study is to maintain the availability of cellular networks in disturbance scenarios. In order to extend the disaster time functionality, energy usage optimization is needed to maintain reasonable coverage and capacity. Simulations performed with different network layouts show the effects of choosing only a portion of evolved node B (eNB) macrosites to operate at a time. Different sets of three to nine threesectored eNB sites are selected to study how the network would perform with a limited number of eNB sites. Simulation results show how the mobile network availability duration can be sustained by selecting a set of eNB sites to operate at a time and still maintain a reasonable service level and availability in disturbance scenarios. An increase of $100 \%$ to $500 \%$ can be achieved in the duration of "backup coverage" in cellular networks with backup batteries when the percentage of active eNB sites is reduced down to $20 \%$.
\end{abstract}

\section{Introduction}

Disaster and disturbance scenarios usually occur without a warning. Whether they are natural weather-based storms or disasters caused by human, such as accidents or sabotage, the effects can be devastating and usually prevent the normal utilization of mobile networks. Typically, storms are the cause of blackouts in electrical grids [1]; furthermore, they have an impact on public safety and commercial mobile networks, thus yielding service and communication outages in urban and rural areas. This can eventually prevent citizens from requesting emergency help in these outage areas. In addition, maintenance and rescue teams can not communicate through commercial mobile networks and have to have a separate communication system.

Service outages in mobile networks are mainly caused by (storm-related) power outages. In order to enable some service in these cases, evolved node B (eNB) macrosites are typically supplied with backup batteries. These reserve energy resources provide power to run eNBs, but only for a limited time period. In Finland, this corresponds to $2-4$ hours, as required by the Finnish Communications Regulatory Authority [2]. After strong weather phenomenon, in the worst case, the repair-work may take several days resulting in the unavailability of commercial cellular networks that may also endanger rescue operations. An alternative is to have aggregates over the network or at certain critical eNB sites, to guarantee their electricity supply for a longer period of time. Because aggregates are slightly costly to be supplied and used at every site, some optimization is needed in a similar way as in the case of battery backups; that is, how many aggregates should be enough to enable sufficient cellular network coverage? Moreover, in case of longer term of electrical cut-offs (i.e., over one day), aggregates are eventually required to guarantee (cost-efficient) mobile network communications in disturbance scenarios.

Another type of critical discontinuity in mobile networks may happen due to major malfunctions in the core transmission network, major damage in the core network elements, such as controllers, or in the switching functions. These malfunctions can also be caused by sabotage or a cyberattack, which may cause very wide discontinuity in the whole mobile 
communications network. However, these are out of the scope of this study as this paper is concentrating more on optimizing the backup energy utilization.

Some solutions for mobile communications have been proposed to manage these disturbance and disaster scenarios to improve the resiliency of mobile networks. In Japan, the so called critical sites have been implemented in urban areas to durable high-rise buildings [3] with at least $24 \mathrm{~h}$ backup batteries to give continuous service, for example, in case of disasters. High altitude platforms (HAPs) [4] have been discussed earlier as a possible solution to provide service coverage in the case where hurricanes and tornadoes have destroyed the existing infrastructure of a cellular network in the disaster area. This idea has been even taken further with Google's project Loon, which targets to provide worldwide Internet access through HAPs, implemented with balloon platforms [5]. Also Facebook has their HAPs approach in their internet.org project [6]. Instead of utilizing balloons, Facebook relies on solar powered drones.

There are also solutions to utilize satellite communication services for disaster areas although these systems are rather expensive and might not work inside buildings. Moreover, temporary movable networks, like mobile ad hoc networks (MANETs), are a popular research area for disaster scenarios as seen from publications on the topic in [7-9]. Many of these solutions are replacing the existing infrastructure with a new one instead of trying to improve it.

In this paper, the aim is to utilize the traditional macrocellular network infrastructure and extend the availability of the network several times from the current backup time when the electrical grid is down and the mobile network relies on backup power. This is possible with an approach, where only a portion of the eNB sites are utilized at a time and switched to another set of sites when the first set runs out of energy. This differs from a traditional sleep mode technique where the nonactive eNBs are not utilized at all during the "sleep time" to save as much energy as possible, which is important in disturbance situations. This will obviously degrade the coverage availability and capacity, but disturbance and disaster scenarios require exceptional methods to enable even some service as long as possible. Only macrosites are considered in this study because small cells are usually available only within cities and they are not equipped with backup power. Maintaining reasonable coverage for disturbance scenarios in both urban and rural areas is emphasized; that is, the focus is on having more resilient mobile networks. Long term evolution (LTE) is utilized in the simulations with evolved UMTS terrestrial radio access (E-UTRA) operating bands 20 and 3 $(800 \mathrm{MHz}$ and $1800 \mathrm{MHz}$ ) for rural environment. E-UTRA bands 3 and $7(1800 \mathrm{MHz}$ and $2600 \mathrm{MHz})$ are utilized for urban environment. All of the considered frequency bands are frequency division duplex (FDD) channels.

\section{Related Work}

This work is related to at least two different energy-saving concepts: cellular networks with sleep modes and green cellular networks. However, the idea of these concepts is not utilized in the traditional way. This paper utilizes the idea of saving energy only in disturbance situations, that is, in disaster scenarios in the following manner: saving energy by utilizing only a portion of the eNB sites to provide "backup coverage" to areas where the electricity supply from the grid has been (temporarily) cut off, for example, due to strong weather phenomenon. Thus, this study goes beyond the state of the art for utilizing mobile network backup energy as this kind of approach has not been proposed for disturbance situations.

The idea of green cellular networks is to have sustainable development in cellular networks towards utilizing sustainable energy sources and taking into account environmental aspects. This usually relates to favouring renewable energy resources to reduce the carbon footprint of cellular network infrastructure or saving energy by reducing the energy consumption of base stations in some manner. Green cellular networks are nowadays studied widely and several publications are available in this field. The authors in [10], for example, present a survey on the state of green cellular networks and the possible challenges they currently have. The largest power consuming part of cellular networks is base stations, as presented in [11]. Furthermore, the largest part of base station power consumption comes from power amplifiers (50-80\%) [12].

The traditional sleep mode concept has been studied, for example, in [13], where the effect of one or more base stations turned to "sleep" is studied. A case study of dynamically switching off base station sites is presented in [14], where the authors show great energy saving in the cellular network during low-traffic periods. One of the most recent wide surveys on these energy-efficient base stations, that is, base station sites with sleep modes as well as green cellular networks, is presented in [15]. The authors present an extensive list of recent publications on these topics and discuss the assumptions and simplifications utilized in these papers to show the great effect they can have in the achievable benefits in actual networks.

Most of the traditional sleep mode techniques consider only a "normal" network utilization. This is because the idea behind most sleep mode techniques is based on saving energy in the low-traffic time periods during the day and usually the sleep modes are only considering these circumstances. This does not necessarily mean that these techniques could not be utilized during disturbance scenarios. However, it should be highlighted that traffic load increases during disturbance scenarios as people try to contact other people from these areas (and vice versa) and utilize the mobile network to search for information regarding the cause of the disturbance situation. Thus, algorithms based on low-traffic network utilization would not work during these events.

A modified sleep mode concept in cellular networks is the main approach utilized in this paper for the functionality of eNB sites operating only with backup power (i.e., during a disturbance scenario). However, during this sleep mode, the eNB sites just wait for their turn to power on, not broadcasting anything during this time. This way, for example, power amplifiers and air conditioning are not needed and signal processing power requirements are very low as there is nothing to transmit or receive during the sleep time. Thus, the power consumption of these "sleeping eNB sites" is very marginal. 


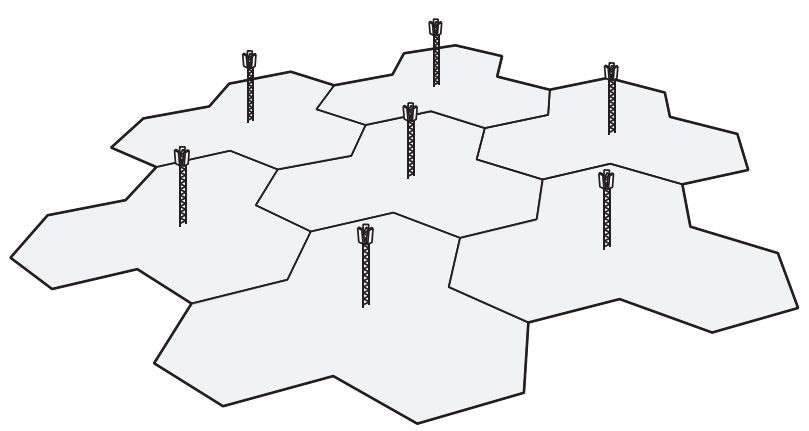

FIGURE 1: Cloverleaf tessellation with seven three-sectored eNB sites.

This is more effective in saving energy than having traditional sleep mode techniques, where the eNBs "wake up" to check the situation every now and then. The approach utilized in the paper does not take into account traffic loads, instead the target is to find out what would be the effect of selecting only a portion of the available eNB sites to the network performance. Moreover, the study does not include algorithms in how to actually select the usable eNB sites (although the selection of eNB sites is based on choosing some eNB sites evenly within a target area).

\section{Radio Network Planning}

Radio network planning is a process to design the best possible wireless communication network such that coverage, capacity, and the quality of service meet (and exceed) the requirements set by the amount of subscribers in a target area. In order to maximize these aspects, a radio network planner needs to pay careful attention to some very important factors. These include the environment, which defines the propagation slope, the model utilized to calculate the maximum path loss, and the network layout in terms of base station locations with respect to other base stations.

3.1. Network Layout. The performance of a cellular network depends on many factors and one of the important factors is the used network layout or more precisely the used tessellation. Ideally, tessellations form a continuous network that has raster-like properties. This means that the layout has symmetric shapes regarding the positions of the eNB sites and antenna directions. The half-power beamwidth (HPBW) of the used antennas also affects the choice of tessellation, but for a 65 degree HPBW antenna the cloverleaf tessellation is the most optimal choice [16]. It is the most commonly used tessellation in Europe and an example of it with seven eNB sites is presented in Figure 1.

Figure 1 shows that every eNB site antenna is pointed directly towards one of the closest neighboring eNB sites. However, this is done in such a way that the antenna beams are pointed in between two other antenna beams thus reducing interference as much as possible.

3.2. Propagation Model. Coverage of a mobile network is defined as a geographical area, where the eNB and user equipment (UE) can still communicate with each other. Coverage estimations can be calculated with reasonable accuracy, for example, by using a well-known Okumura-Hata prediction model equation (or its extended version, the COST-Hata model) [17]:

$$
\begin{aligned}
L= & A+B \cdot \log _{10}\left(f_{\mathrm{MHz}}\right)-13.82 \cdot \log _{10}\left(h_{\mathrm{eNB}}\right) \\
& -a\left(h_{\mathrm{UE}}\right)+\left(C-6.55 \cdot \log _{10}\left(h_{\mathrm{eNB}}\right)\right) \cdot \log _{10}\left(d_{\mathrm{km}}\right) \\
& +C_{m},
\end{aligned}
$$

where $L$ is path loss in $[\mathrm{dB}], A, B$, and $C$ are constants, $f_{\mathrm{MHz}}$ is the used frequency in $[\mathrm{MHz}], h_{\mathrm{eNB}}$ is eNB antenna height in $[\mathrm{m}], h_{\mathrm{UE}}$ is UE antenna height in [m], $a\left(h_{\mathrm{UE}}\right)$ is a city size dependent function, $d_{\mathrm{km}}$ is distance betweene NB and UE in $[\mathrm{km}]$, and $C_{m}$ is an area correction factor.

Before the Okumura-Hata model can be used to get the maximum cell distance, some link budget calculations are needed to define the maximum path loss between the eNB and UE.

It should be noted that the Okumura-Hata model (1) has to be tuned for every environment separately to get more accurate results. In addition, the area correction factor, $C_{m}$, has to be set regarding the propagation environment morphological values. Correspondingly, the propagation slope, which is defined by $\left(C-6.55 \cdot \log _{10}\left(h_{\mathrm{eNB}}\right)\right)$, has to be set regarding the eNB antenna height and the wanted propagation path loss exponent by tuning the $C$ constant.

3.3. Planning Thresholds. Planning thresholds are used together with propagation prediction models to calculate the maximum path loss in the link budget. The main planning threshold value is the slow fading margin [18]. It is calculated from the standard deviation of the slow fading, the propagation slope, and the coverage probability for the service.

It should also be addressed whether the radio network coverage is designed for outdoor or for indoor usage. When designing a radio network with outdoor macrocells, and there should also be some indoor coverage, some additional planning thresholds are needed. First, the value of slow fading margin should be increased and then the building penetration loss should be added to the link budget calculation in order to expect more realistic predictions.

One example of a typical average distance between neighboring eNB sites, that is, intersite distance (ISD), is $750 \mathrm{~m}$ in urban and $7500 \mathrm{~m}$ in rural environment [19]. While maintaining the antenna height and moving eNB sites closer to each other, more coverage overlapping occurs. This means that areas with coverage from one eNB site have coverage also from other neighbor eNB sites. In rural areas, the coverage overlapping is minimized because of large ISDs and, respectively, in urban areas there is more coverage overlapping because of shorter ISDs; that is, the eNB sites are close to each other. This means that the possibilities to decrease the number of eNB sites and still have available coverage should be greater in urban areas than in rural areas, but this depends heavily on the configuration of the network.

3.4. Practical Capacity Requirements. Mobile network capacity requirements depend on population density and the 


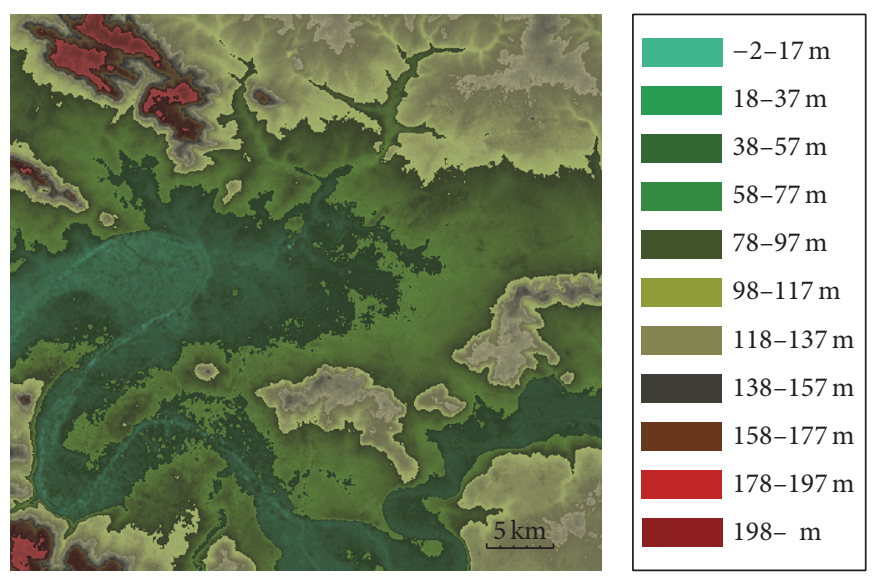

FIGURE 2: Digital elevation map of the simulation area.

requested services. Since mobile network cells are shared mediums, the available capacity per UE depends on the total number of mobile network subscribers within the serving cell and the data throughput each UE requests. To expand this limited capacity, more channels, that is, additional frequency bands, can be utilized together with different technologies. Thus, recent implementations include several frequency layers (bands) and technologies at one eNB site [20]. An example of this would be a base station site that has a GSM base station, a UMTS base station, and a LTE base station (eNB) and each of these technologies would operate on at least two different frequency bands. These could be, for example, GSM operating at $900 \mathrm{MHz}$ and $1800 \mathrm{MHz}$, UMTS operating at $900 \mathrm{MHz}$ and $2100 \mathrm{MHz}$, and LTE operating at $800 \mathrm{MHz}$ and $2600 \mathrm{MHz}$. To maximize availability and thus to minimize the required electrical power, several technologies, for example, GSM and UMTS, and several frequency layers should be switched off (e.g., only the lowest frequency band of the remaining technology is utilized for service, such as LTE $800 \mathrm{MHz}$ ) and still certain services such as short messages, speech, or limited data services should be maintained (depending on the available technology and its capabilities). This results in conserving backup power in crisis situations but still enables services for subscribers for a longer period of time compared with a situation where eNB sites would continue to operate normally and quickly consume the available reserve energy.

\section{Simulations}

Link-level simulations were implemented using commercially available radio network planning software called ICS Designer. The area was a randomly picked and relatively flat area in France, which had a $25 \mathrm{~m}$ resolution digital elevation map as shown in Figure 2. After the simulation results were obtained, they were exported into Matlab software for further analysis and visualization.

4.1. Simulation Setup and Environment. All simulations were based on a macrocellular cloverleaf tessellation with eNB sites that had three sectors. A conventional eNB grid of 19 sites was used as a reference case, which has one eNB site in the middle and two tiers, first tier with six sites and second tier with twelve eNB sites, around it forming a hexagonal grid. All eNB sites in the original layout had an equal ISD of $500 \mathrm{~m}$ for urban environment and $4000 \mathrm{~m}$ for rural environment. Circular areas with $1.25 \mathrm{Km}$ and $10 \mathrm{Km}$ radii from the centre eNB site were the base "area of interest" for the analysis. In total 57 cells were the maximum amount of cells in one area. In order to take into account sites outside the target area, three more tiers of eNB sites were placed around the central grid of eNB sites. Thus, continuous coverage was formed such that the selection of eNB sites was repeated around the target area to avoid errors in the edge areas of the calculation area.

The antenna height values of $20 \mathrm{~m}$ and $40 \mathrm{~m}$ with fivedegree antenna tilting were used in the simulations. The horizontal antenna HPBW was 65 degrees with $17.22 \mathrm{dBi}$ gain for eNB sites while UE antennas were omnidirectional antennas with $0 \mathrm{dBi}$ gain.

Okumura-Hata model was used for calculating the path loss values in the simulations. Fading was taken into account with log-normal distribution having zero mean and $9 \mathrm{~dB}$ standard deviation. All key parameters regarding the simulations are presented in Table 1. The distribution of transmit power to different LTE downlink physical channels is visible in Table 2.

4.2. Simulation Scenarios. Simulation scenarios had a large number of different types of network layouts. The idea was to find suitable configurations to achieve good enough coverage for the simulation area and at the same time minimize the service outage. The cellular network can function for an extended period of time when only a part of the network is working on backup energy at a time. In order to reduce the number of different possible combinations which would be possible for a set of eNB sites, the number of different kinds of sets out of all possible sets was reduced. If all possible combinations would be considered, the maximum number of different sets (in any order), for example, with nine eNB sites out of 19 eNB sites would be $\left(\begin{array}{c}19 \\ 9\end{array}\right)=92378$, and even three eNB sites out of 19 eNB sites would have $\left(\begin{array}{c}19 \\ 3\end{array}\right)=969$ different sets. Thus, the number of different sets was set at a small amount, 
TABLE 1: Key simulation parameters.

\begin{tabular}{lcc}
\hline Parameter & Value & Unit \\
\hline Operating frequency band 20 (FDD) & 800 & $\mathrm{MHz}$ \\
Operating frequency band 3 (FDD) & 1800 & $\mathrm{MHz}$ \\
Operating frequency band 7 (FDD) & 2600 & $\mathrm{MHz}$ \\
\hline Bandwidth & 10 & $\mathrm{MHz}$ \\
Number of usable resource blocks & 50 & $\mathrm{pcs}$ \\
Loading & 75 & $\%$ \\
\hline Calculation resolution & 25 & $\mathrm{~m}$ \\
Intersite distance, urban & 500 & $\mathrm{~m}$ \\
Intersite distance, rural & 4000 & $\mathrm{~m}$ \\
\hline Antenna height, urban & 20 & $\mathrm{~m}$ \\
Antenna height, rural & 40 & $\mathrm{~m}$ \\
Building height & 8 & $\mathrm{~m}$ \\
\hline eNB antenna HPBW & 65 & $\circ$ \\
eNB antenna gain & 17.22 & $\mathrm{dBi}$ \\
Additional losses & 3 & $\mathrm{~dB}$ \\
\hline UE antenna height & 1.5 & $\mathrm{~m}$ \\
UE antenna gain & 0 & $\mathrm{dBi}$ \\
\hline Max. eNB TX power & 20 & $\mathrm{~W}$ \\
\hline
\end{tabular}

TABLE 2: Distribution of downlink transmit power to different LTE physical channels.

\begin{tabular}{lcc}
\hline LTE physical channel & Share & Unit \\
\hline Reference signal & 4.76 & $\%$ \\
Physical downlink shared channel (PDSCH) & 74.33 & $\%$ \\
Physical downlink control channel (PDCCH) & 20.24 & $\%$ \\
Physical broadcast channel (PBCH) & 0.33 & $\%$ \\
Primary synchronization signal (P-SS) & 0.17 & $\%$ \\
Secondary synchronization signal (S-SS) & 0.17 & $\%$ \\
\hline Total & 100 & $\%$ \\
\hline
\end{tabular}

six in this paper, to study how the performance of the network behaves as a function of available eNB sites. The focus is especially to study having less than half of the eNB sites per studied area. The eNB sites chosen for a set were based on spreading the available sites as evenly as possible to the studied area, since, for example, choosing only the three closest eNB sites from one "corner" of the grid would results in the uneven distribution of backup coverage in the target area of interest.

Figure 3 shows how eNB sites were chosen for three to nine eNB site cases. As can be seen, the eNB sites were chosen in a way to be able to serve the target area as evenly as possibly. An example of service probability with two different sets of six eNB sites is visualized in Figure 4.

\section{Results}

Figure 5 shows the mean reference signal received power (RSRP) level, Figure 6 the mean service probability, and Figure 7 the average signal-to-interference-and-noise ratio (SINR) with respect to the number of eNB sites. Figures 8 and 9 present the average area throughput to study the available capacity with respect to the amount of eNB sites. The corresponding exact values are presented in Tables 3 and 4 .

The mean RSRP values in Figure 5 show that as the number of eNB sites grow the average RSRP increases, which is expected. Rural environment with E-UTRA band $3(1800 \mathrm{MHz})$ has the lowest average RSRP values and correspondingly rural environment with E-UTRA band 20 $(800 \mathrm{MHz})$ has the highest RSRP values. The average values of RSRP are clearly different for all cases. First of all, the environment type with different antenna heights for the rural and urban areas has a huge impact on the average RSRP values, since the antenna height for the rural area is two times the height of the urban area. Next, another important factor is the utilized frequency; that is, for rural area, the higher frequency band is more than twice the utilized lower frequency band. In the urban cases, the higher frequency band is less than twice the utilized lower frequency band; thus, the differences between the average RSRP values for the two rural cases is more dramatic than the corresponding differences for the two urban cases. Finally, the urban case is also affected by the buildings, thus lowering the average RSRP values. In this study, the lowest value was $-82.68 \mathrm{dBm}$ with $15.79 \%$ of eNB sites available.

Figure 6 presents the average probability of having backup coverage in the different cases. The lowest probability of having service coverage was with E-UTRA band $7(2600 \mathrm{MHz})$ in the urban environment. The lowest value was $55.44 \%$ with $15.79 \%$ of the eNB sites available. Thus, although the urban area had denser eNB site placement than the rural area, the coverage areas with a higher frequency band are quite limited as well as overlapping areas. This is mostly because the attenuation is higher in the urban environment (with buildings) as well as in a higher frequency band. When the same frequency band was utilized in both the urban and rural environment, the urban E-UTRA band 3 had slightly higher probability of having a service than its rural counterpart (rural E-UTRA band 3). The highest service availability was achieved with the lowest frequency, E-UTRA band 20 in the rural case, where the probability of having a service was over $80 \%$ even with the lowest number of eNB sites (15.79\%). In order to have over $80 \%$ probability of (backup) coverage in other cases, E-UTRA band 3 cases $(1800 \mathrm{MHz})$ would need around $26.32 \%$ in urban and $31.58 \%$ in rural scenarios and E-UTRA band 7 $(2600 \mathrm{MHz})$ around $50 \%$ in the urban environment.

When the exact values are observed from Table 3 as a function of available eNB sites, the differences between different E-UTRA bands and environments are easy to compare as well as the differences between each parameter. Intuitively thinking, each parameter (RSRP, service probability, and SINR) should have a better value as the percentage of eNB sites per area is increased and in general this is observed in Table 3. The only difference is the utilized lower urban frequency band $(1800 \mathrm{MHz})$, where the average SINR value is slightly increased when more than $31.58 \%$ of eNB sites are utilized. However, this increase stops after more than $42.11 \%$ of eNB sites are utilized.

In Figure 7, the mean SINR values are presented. It can be noticed that, in rural environment, the achievable values are higher than in urban cases, mostly because of low interference 


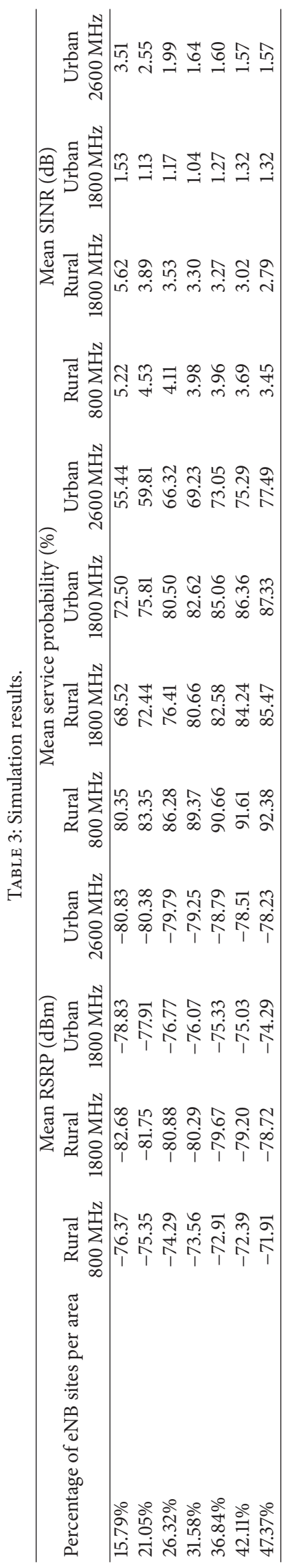


TABLE 4: Mean area throughput.

\begin{tabular}{|c|c|c|c|c|}
\hline \multirow{3}{*}{ Percentage of eNB sites per area } & \multicolumn{4}{|c|}{ Mean area throughput $\left(\mathrm{Mbps} / \mathrm{km}^{2}\right)$} \\
\hline & Rural & Rural & Urban & Urban \\
\hline & $800 \mathrm{MHz}$ & $1800 \mathrm{MHz}$ & $1800 \mathrm{MHz}$ & $2600 \mathrm{MHz}$ \\
\hline $15.79 \%$ & 0.23 & 0.24 & 9.07 & 11.79 \\
\hline $21.05 \%$ & 0.28 & 0.26 & 11.44 & 13.88 \\
\hline $26.32 \%$ & 0.33 & 0.31 & 14.38 & 16.11 \\
\hline $31.58 \%$ & 0.39 & 0.36 & 16.94 & 18.43 \\
\hline $36.84 \%$ & 0.46 & 0.42 & 20.40 & 21.38 \\
\hline $42.11 \%$ & 0.50 & 0.46 & 23.49 & 24.32 \\
\hline $47.37 \%$ & 0.55 & 0.50 & 26.43 & 27.37 \\
\hline
\end{tabular}

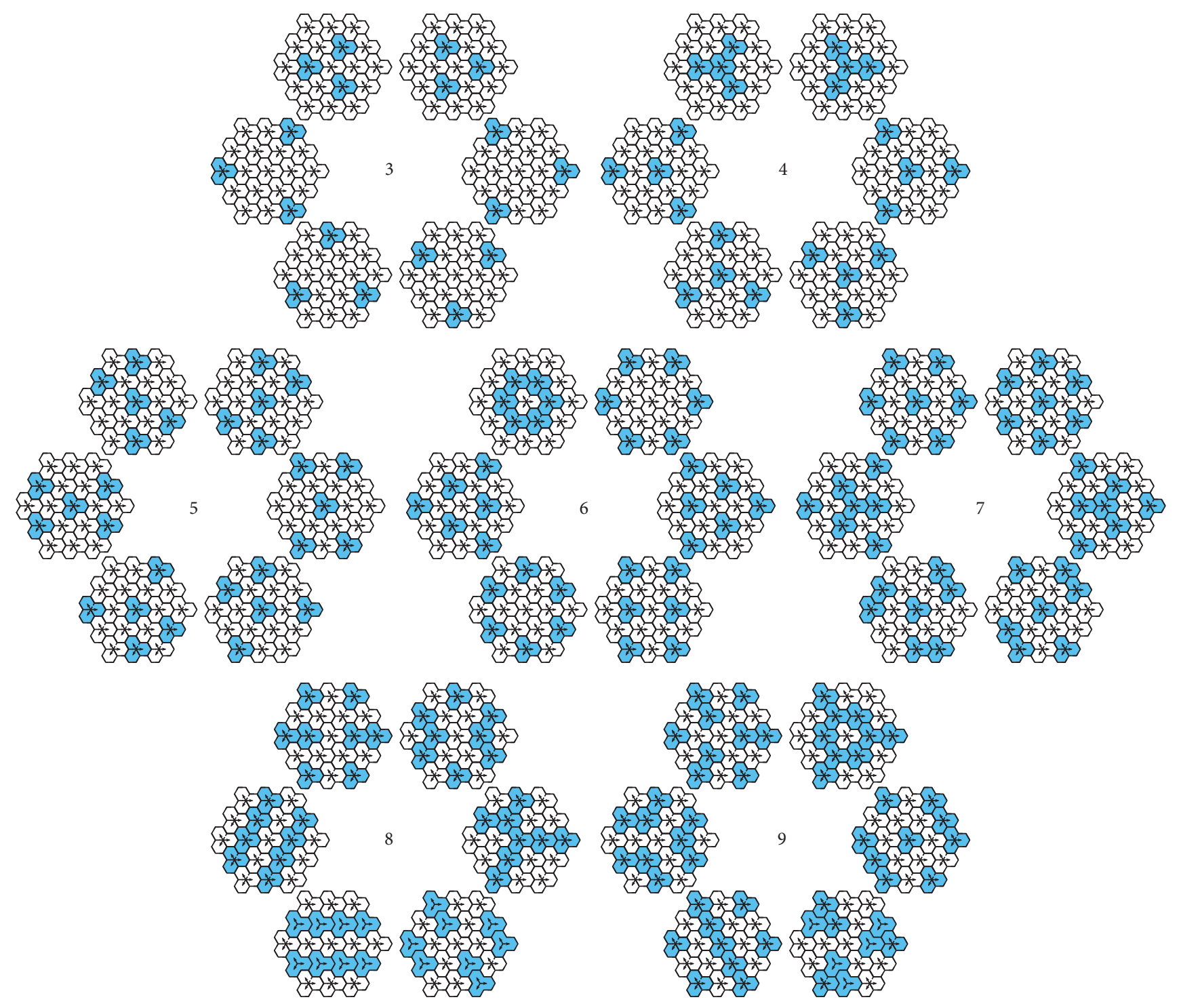

FIGURE 3: Selection of different three to nine eNB site configurations that were utilized in the simulations for a target area (and repeated in a similar way around this area to form a continuous coverage). The selected eNB sites are highlighted with blue color. 

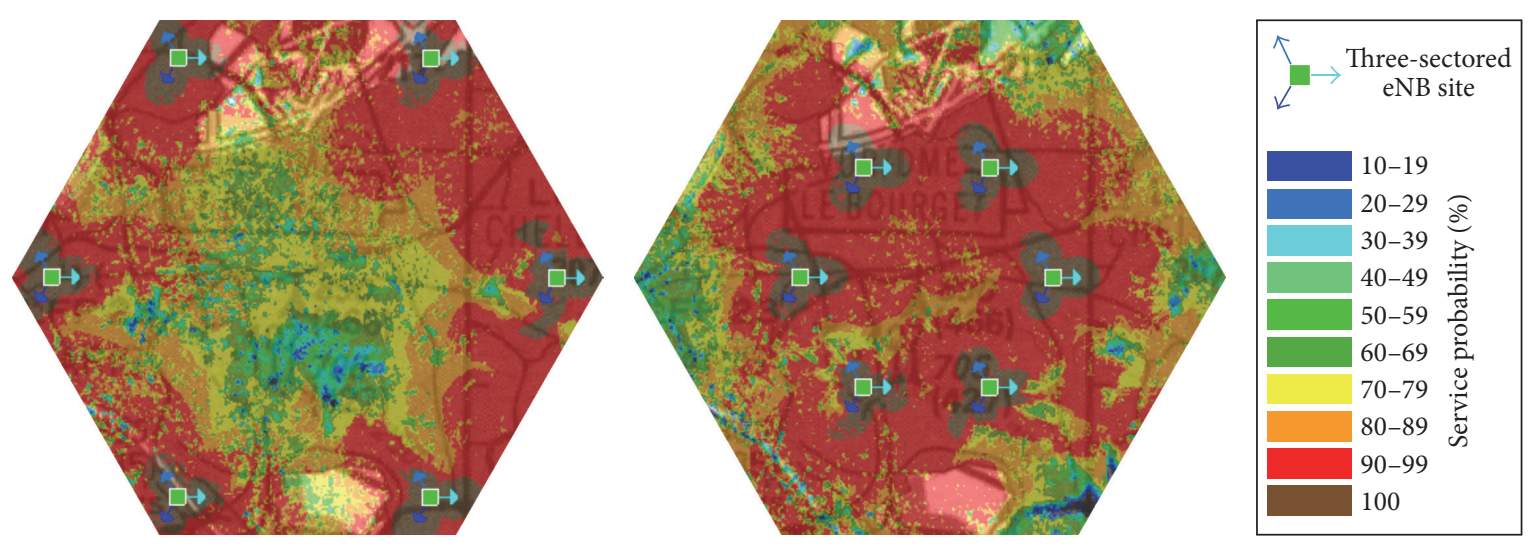

FIGURE 4: An example of service availability with two different sets of six eNB sites.

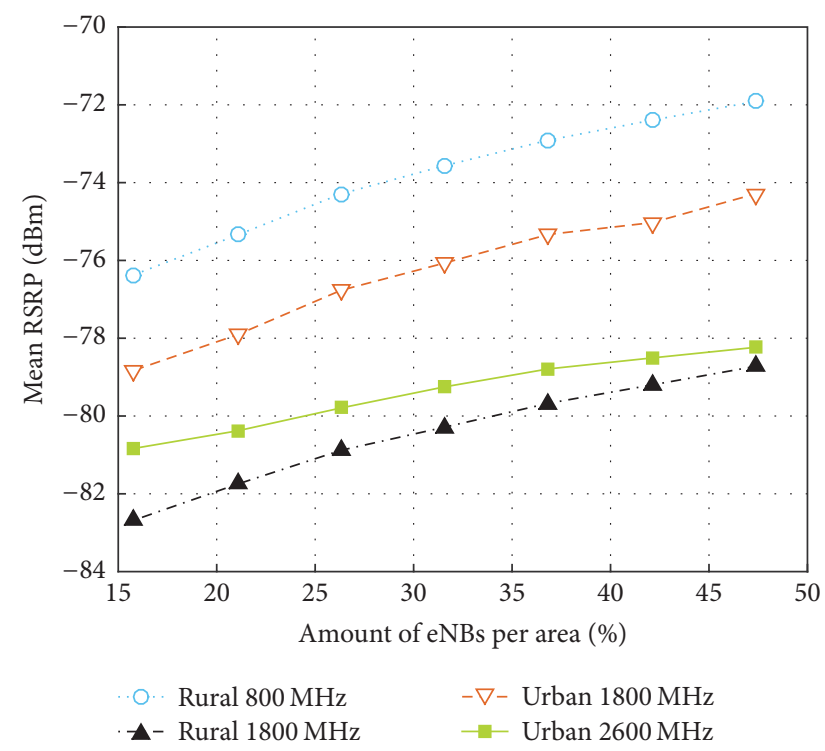

FIGURE 5: Average RSRP level with respect to the amount of eNB sites per area.

from less dense eNB site placements; that is, the minimum ISD is $4000 \mathrm{~m}$. When more eNB sites are available, the average SINR degrades because of the increased interference levels.

Figures 8 and 9 show the average throughput per area. As can be noted, the available average data rates are much higher in urban cases since the ISD is much lower; that is, the network is much denser. It should be remembered that the loading of the network was set to $75 \%$; thus only 37 resource blocks were available out of 50 resource blocks available in the $10 \mathrm{MHz}$ bandwidth for LTE.

\section{Conclusions and Discussion}

In this paper, the availability of mobile networks in disturbance scenarios was studied by simulating different network layouts for macro-eNB sites. Thus, a solution for improving the cellular network functionality in disturbance situations was presented, which is a very important topic in the

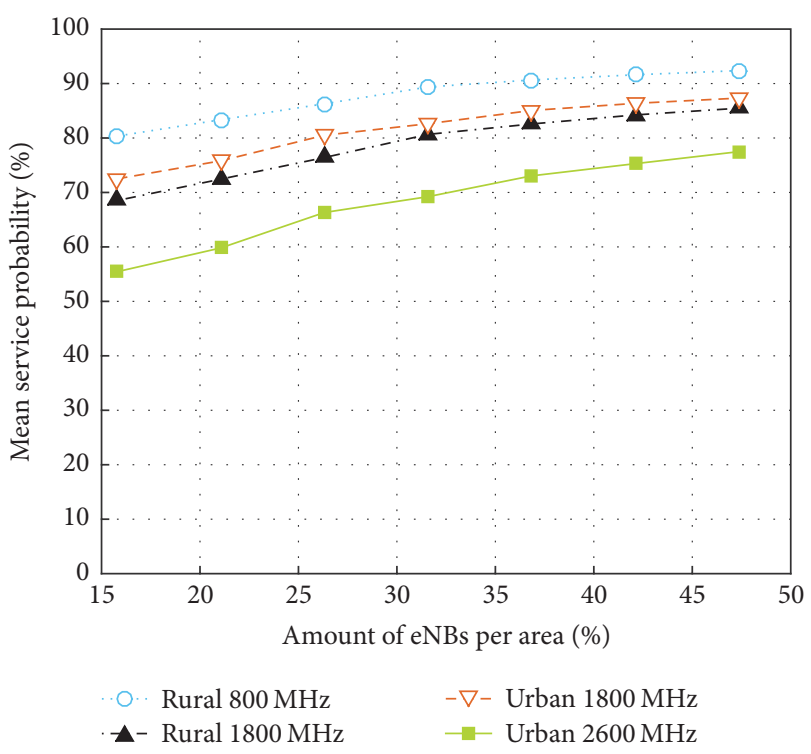

FIGURE 6: Average service probability with respect to the amount of eNB sites per area.

resilience of mobile networks. The target of the simulations was to find out how a limited network configuration would perform in terms of coverage and capacity with mobile network availability (without adding additional backup power sources). This minimum configuration can be utilized with battery backups to extend the network availability called backup coverage. As the name suggests, this exceptional state of the network cannot have "normal" service availability as the goal is to provide "acceptable" or at least some coverage that would last much longer than initially. This would mean that some areas would not have coverage at every time instant during the backup coverage time window, but since the operating sets of eNB sites are cycled over time, all areas would have a certain time window when the service is still available. Thus, only $20 \%$ to $42 \%$ of eNB sites are needed in order to achieve approximately $75 \%$ availability in urban areas. Correspondingly, $15 \%$ to $25 \%$ of eNB sites are needed in rural areas for the same availability. This means that 


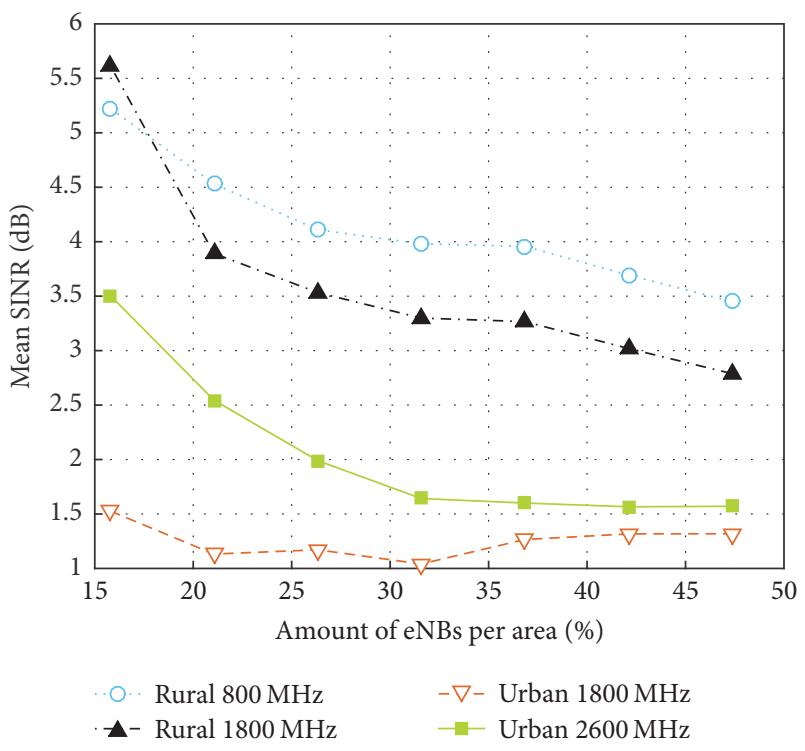

FIGURE 7: Average SINR with respect to the amount of eNB sites per area.

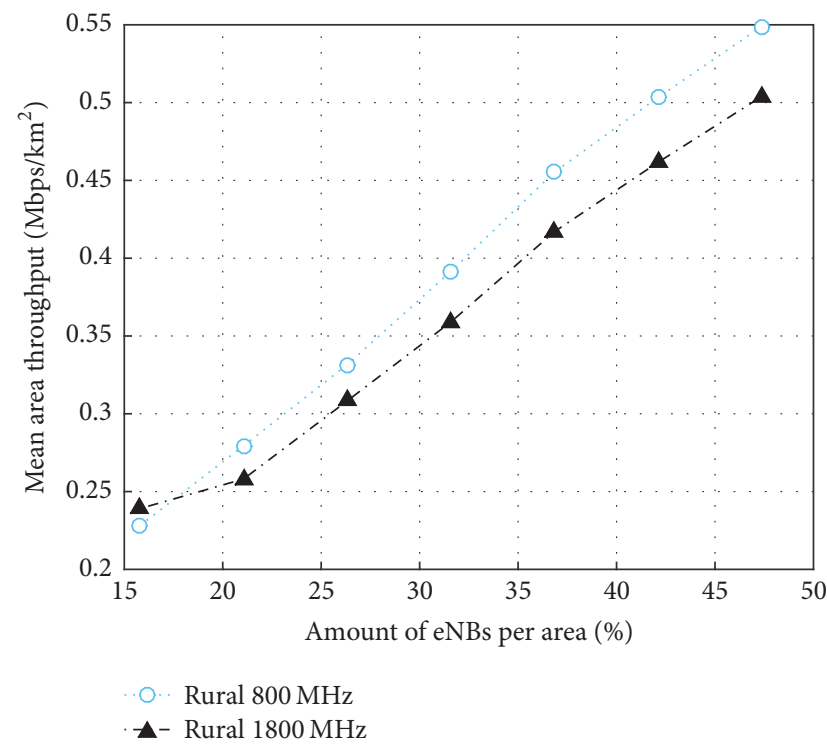

FIGURE 8: Average area throughput with respect to the amount of eNB sites per area in rural environment.

an increase of $100 \%$ to $500 \%$ could be achieved on the operational time of backup coverage, correspondingly. This also translates to having mediocre coverage for a longer period of time if approximately $20 \%$ of eNB sites would be equipped with aggregates or any other means of powering up eNB sites, which would be cheaper than having, for example, $24 \mathrm{~h}$ backup batteries deployed to every possible eNB site.

The comparison of the achieved results with other sleep mode techniques is not that straightforward. First of all, the traditional sleep mode techniques are designed to save energy during low-traffic time periods and this is not the case during disturbance situations. Second, many studies such as [13-15] consider different sleep mode techniques with different kinds

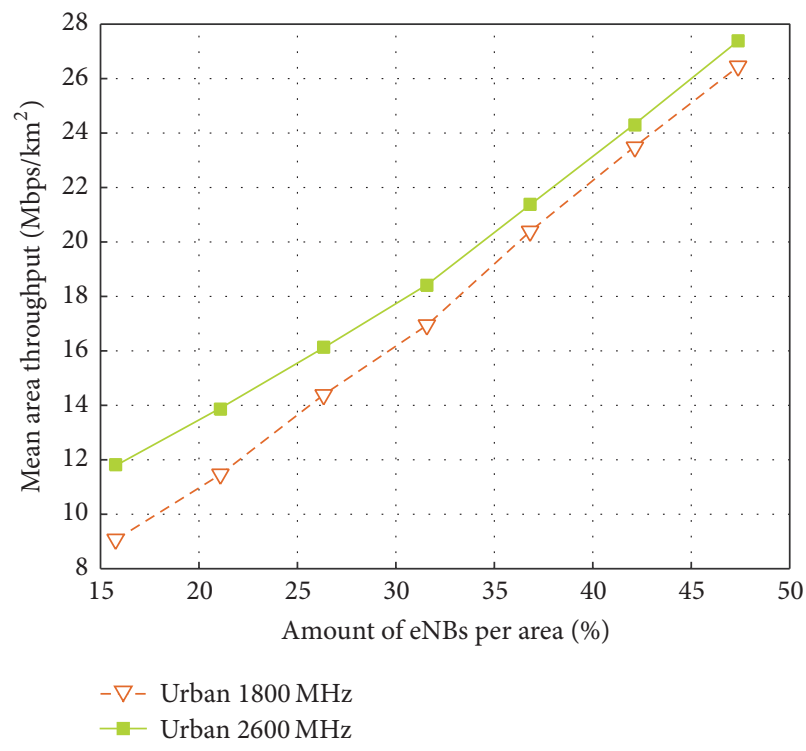

FIGURE 9: Average area throughput with respect to the amount of eNB sites per area in urban environment.

of eNB site layouts with heterogeneous networks (besides macro eNB sites, there can also be micro eNB sites and small cell eNB sites). In order to get some idea about the effectiveness of the approach utilized within this paper, the authors in [21] present a comparable sleep mode technique, where part of the eNB sites remains active all the time and part of the eNB sites can switch to sleep mode. The results in the study suggest that on average the power savings from an actual network implementation would be from $7.38 \%$ to $27.72 \%$ on a $24 \mathrm{~h}$ time period. Thus, although still not directly comparable, the energy savings are clearly a lot less than in the approach utilized in this study. The difference is mostly explained with the fact that the sleep mode technique is designed to keep high quality of service (QoS), that is, high service availability. In this paper the requirements for QoS are clearly lower since the idea is lower QoS to enable even some sufficient service level for a clearly longer period of time by aggressively saving energy.

Although it seems that there would be more overlapping in the rural areas, it should be noticed that the service availability is higher in urban areas when the same E-UTRA frequency bands are utilized (band 3). This indicates that the operational time of the backup coverage in urban areas would last somewhat longer. Thus, the achievable gain is strongly related to the overlapping rate of neighboring cells which is also defined by the utilized frequency band as well as the configuration of the eNB sites.

The studied availability can be reached in existing networks by utilizing existing configurations and resources by adding only controlling units. Moreover, the achieved results assume that transmission lines and core networks are properly functioning (which is usually the case, at least in Finland, as the backhaul element backup power regulation is stricter than for eNB sites by a factor of 2-6 [2]), and thus more specific solutions need to be proposed to maintain their 
availabilities in case of disconnections. It should be noted that, in real operational networks, the selection of eNB sites is not so straightforward. The geographical distribution of eNB sites is not symmetrical and only roughly follows the cloverleaf tessellation. Moreover, this study only showed how the network would perform if each eNB site was independent of another eNB site; that is, the study did not take into account the possible backhaul connection limitations of linked eNB sites. This would have more serious effects within rural environments where wireless microwave links are utilized more frequently compared with urban areas, where wireless links between eNB sites rarely exist. The results are valid only for the given configuration; however, they were chosen so they represent possible real-life implementations and provide insight on the level of service availability with a limited cellular network configuration.

The future work on this topic will consider more practical eNB site layouts; that is, the distribution of sites will be closer to real-life implementations with more clustered locations of eNB sites. Moreover, the effect of wireless microwave links, that is, the backhaul connection, will also be taken into account based on an operational cellular network operator.

\section{Competing Interests}

The authors declare that they have no competing interests.

\section{Acknowledgments}

Author Joonas Säe would like to thank Tuula and Yrjö Neuvo Fund and Finnish Foundation for Technology Promotion for supporting the research work. The authors would also like to thank European Communications Engineering (ECE) Ltd. and the Finnish Funding Agency for Innovation (TEKES) for funding the research work.

\section{References}

[1] J. Strandén, H. Krohns, P. Verho, and J. Sarsama, "Major disturbances-development of preparedness in finland during the last decade," in Proceedings of the 21st International Conference on Electricity Distribution, p. 4, Frankfurt, Germany, June 2011.

[2] FICORA 54 B/2014 M, "Määräys viestintäverkkojen ja-palvelujen varmistamisesta sekä viestintäverkkojen synkronoinnista," December 2014 (Finnish), https://www.viestintavirasto .fi/attachments/maaraykset/Viestintavirasto54B2014M.pdf.

[3] NTT DoCoMo, "Deployment status of the new disaster preparedness measures," 2012, https://www.nttdocomo.co.jp/english/info/media_center/pr/2012/pdf/20120223_attachment02.pdf.

[4] P. Lähdekorpi, T. Isotalo, K. Kylä-Liuhala, and J. Lempiäinen, "Replacing terrestrial UMTS coverage by HAP in disaster scenarios," in Proceedings of the European Wireless Conference (EW '10), pp. 14-19, Lucca, Italy, April 2010.

[5] Google, "Loon for all," http://www.google.com/loon/.

[6] Internet.org by Facebook, https://info.internet.org/en/story/ connectivity-lab/.

[7] M. Conti and S. Giordano, "Mobile ad hoc networking: milestones, challenges, and new research directions," IEEE Communications Magazine, vol. 52, no. 1, pp. 85-96, 2014.
[8] L. Sassatelli, A. Ali, M. Panda, T. Chahed, and E. Altman, "Reliable transport in delay-tolerant networks with opportunistic routing," IEEE Transactions on Wireless Communications, vol. 13, no. 10, pp. 5546-5557, 2014.

[9] A. Polydoros, N. Dimitriou, G. Baldini, I. N. Fovino, M. Taddeo, and A. M. Cipriano, "Public protection and disaster relief communication system integrity: a radio-flexibility and identity-based cryptography approach," IEEE Vehicular Technology Magazine, vol. 9, no. 4, pp. 51-60, 2014.

[10] Z. Hasan, H. Boostanimehr, and V. K. Bhargava, "Green cellular networks: a survey, some research issues and challenges," IEEE Communications Surveys and Tutorials, vol. 13, no. 4, pp. 524540,2011

[11] C. Han, T. Harrold, S. Armour et al., "Green radio: radio techniques to enable energy-efficient wireless networks," IEEE Communications Magazine, vol. 49, no. 6, pp. 46-54, 2011.

[12] L. M. Correia, D. Zeller, O. Blume et al., "Challenges and enabling technologies for energy aware mobile radio networks," IEEE Communications Magazine, vol. 48, no. 11, pp. 66-72, 2010.

[13] M. A. Marsan, L. Chiaraviglio, D. Ciullo, and M. Meo, "On the effectiveness of single and multiple base station sleep modes in cellular networks," Computer Networks, vol. 57, no. 17, pp. 32763290, 2013.

[14] E. Oh, B. Krishnamachari, X. Liu, and Z. Niu, "Toward dynamic energy-efficient operation of cellular network infrastructure," IEEE Communications Magazine, vol. 49, no. 6, pp. 56-61, 2011.

[15] J. Wu, Y. Zhang, M. Zukerman, and E. K.-N. Yung, "Energyefficient base-stations sleep-mode techniques in green cellular networks: a survey," IEEE Communications Surveys and Tutorials, vol. 17, no. 2, pp. 803-826, 2015.

[16] J. Itkonen, B. Tuzson, and J. Lempiainen, "Assessment of network layouts for CDMA radio access," EURASIP Journal on Wireless Communications and Networking, vol. 2008, Article ID 259310, 11 pages, 2008.

[17] D. Cichon and T. Kümer, "Propagation prediction models," in Digital Mobile Radio Towards Future Generation Systems-Final Report, COST Action 231, Ed., chapter 4, pp. 115-208, European Commission, Tech. Rep, 1999.

[18] J. Lempiäinen and M. Manninen, UMTS Radio Network Planning, Optimization and Qos Management: For Practical Engineering Tasks, Kluwer Academic, Norwell, Mass, USA, 2004.

[19] 3GPP, “Technical specification group radio access network; UMTS $900 \mathrm{MHz}$ work item technical report (Release 8)," 3rd Generation Partnership Project (3GPP), Tech. Rep. TR 25.816, 2009.

[20] Nokia, "Flexi multiradio 10 base station," http://networks.nokia .com/sites/default/files/document/nokia_flexi_multiradio_10_ base_station_brochure_0.pdf.

[21] A. Ali and S. E. Elavoubi, "Design and performance evaluation of site sleep mode in LTE mobile networks," in Proceedings of the 26th International Teletraffic Congress (ITC '14), pp. 1-6, September 2014. 

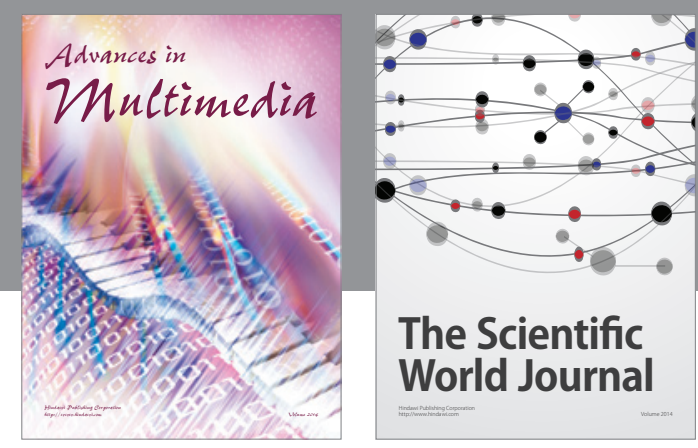

The Scientific World Journal
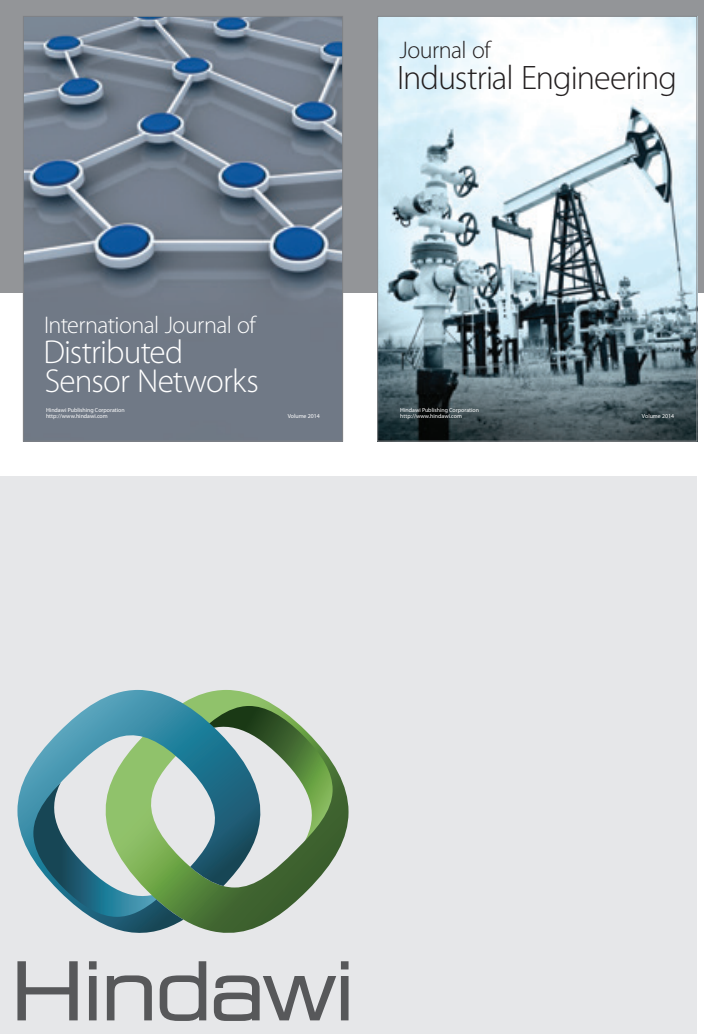

Submit your manuscripts at

http://www.hindawi.com

\section{Computer Networks} and Communications
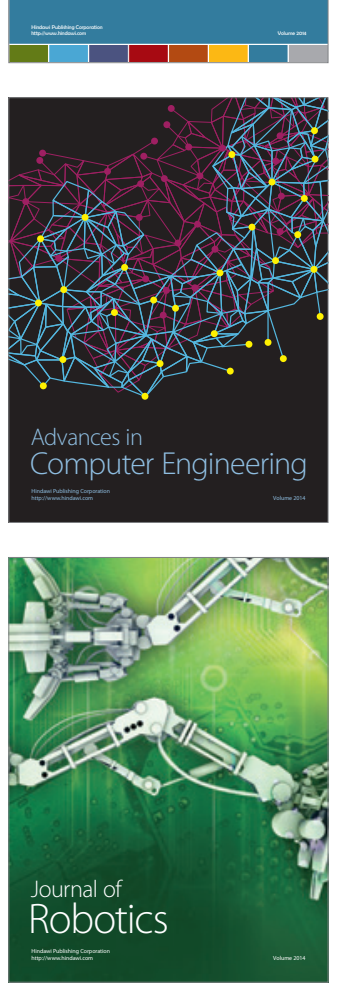
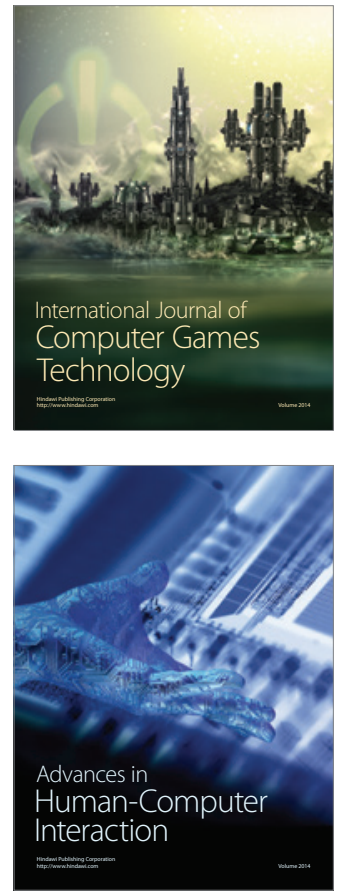
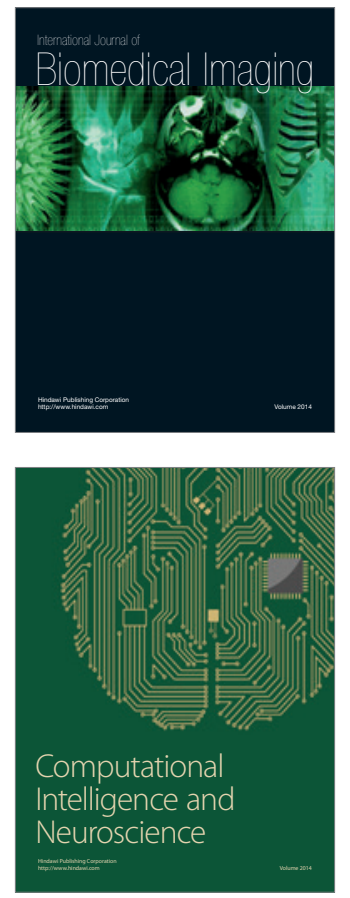
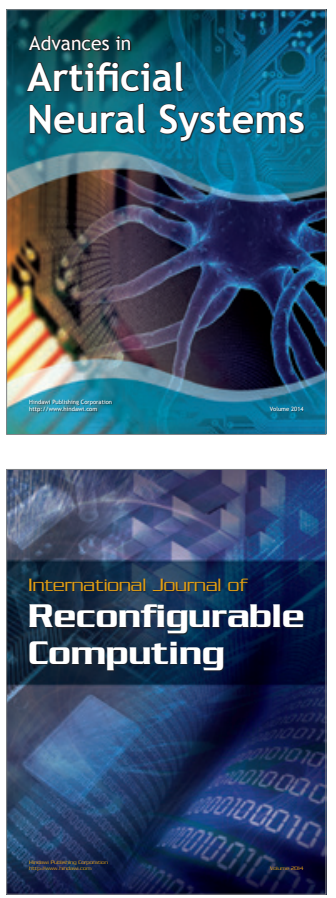
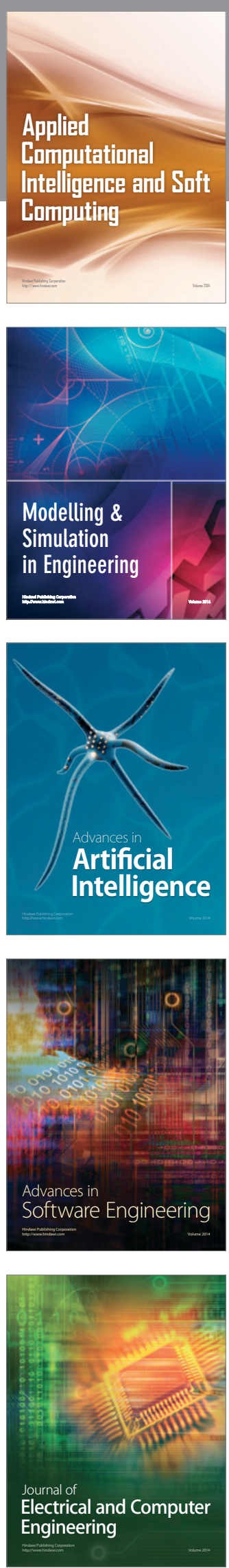